\title{
Estudo Comparativo entre Traqueostomia Precoce e Tardia em Pacientes sob Ventilação Mecânica*
}

\author{
A Comparative Study between Early and Late \\ Tracheostomy in Patients ongoing Mechanical Ventilation
}

Sylvia Carolina Aranha1', Sergio Elia Mataloun², Marcelo Moock ${ }^{3}$, Ricardo Ribeiro ${ }^{4}$

\section{RESUMO}

JUSTIFICATIVA E OBJETIVOS: O objetivo deste estudo foi calcular a incidência de traqueostomia em pacientes submetidos à ventilação mecânica invasiva (VMI) e comparar o tempo de internação, de VMI, APACHE II e a mortalidade entre os pacientes submetidos à traqueostomia, segundo o momento de sua realização (precoce ou tardia).

MÉTODO: Através de estudo retrospectivo observacional tipo coorte, avaliou-se, de abril a outubro de 2005, 190 pacientes internados na UTI adulto do Hospital Estadual do Grajaú.

RESULTADOS: Dos 190 pacientes, 32 (16,84\%) foram submetidos à traqueostomia, permanecendo maior período na unidade de terapia intensiva (UTI) $(30,16$ dias) quando comparados àqueles que não o foram ( $p$ $<0,001)$. A média do tempo de realização da traqueostomia foi de 13,5 dias de início da ventilação mecânica

1. Médica Residente de Clínica Médica da Faculdade de Medicina da Universidade de Santo Amaro.

2. Professor Adjunto da Disciplina de Medicina Intensiva da Faculdade de Medicina da Universidade de Santo Amaro.

3. Professor Adjunto e Chefe da Disciplina de Medicina Intensiva da Faculdade de Medicina da Universidade de Santo Amaro; Chefe da Unidade de Terapia Intensiva do Hospital Estadual do Grajaú.

4. Médico Pediatra do Centro de Saúde Escola da Faculdade de Medicina da Universidade de Santo Amaro.

*Recebido da Unidade de Terapia Intensiva do Hospital Estadual do Grajaú, São Paulo, SP

Apresentado em 13 de fevereiro de 2007

Aceito para publicação em 07 de novembro de 2007

Endereço para correspondência:

Dra. Sylvia Carolina Aranha

Rua Vicente Oropallo, 70/53

05351-025, São Paulo, SP

Fones: (11) 3719-4336 - 8393-5269

E-mail: sylvia_aranha@yahoo.com.br

(C)Associação de Medicina Intensiva Brasileira, 2007 invasiva (VMI). Sabe-se que ainda não existem diretrizes para o estabelecimento do período ideal de sua realização. Neste estudo, a prevalência de traqueostomia precoce ( $\leq 13$ dias) foi $46,87 \%(n=15)$ e tardia $(>13$ dias) $53,13 \%(n=17)$. De maneira significativa, os pacientes com traqueostomia precoce obtiveram escore APACHE II superior àqueles com traqueostomia tardia $(18,2$ versus 13,47$)$, no entanto, não houve diferença entre eles quanto à taxa de mortalidade, devendo-se lembrar que este índice leva em consideração apenas as primeiras $24 \mathrm{~h}$. Não houve diferenças quanto aos tempos de internação na UTI (28,9 versus 31,28 dias) e VMI $(29,73$ versus 32,23 dias) nos dois grupos.

CONCLUSÕES: A incidência de traqueostomia foi elevada, associando-se a menor mortalidade na UTI, mas com maior duração da internação e de complicações. Não houve diferença significativa quanto ao destino e tempo de VMI dos pacientes quando submetidos à traqueostomia precoce ou tardia.

Unitermos: traqueostomia, UTI, ventilação mecânica

\section{SUMMARY}

BACKGROUND AND OBJECTIVES: To assess the incidence of tracheostomy in patients submitted to mechanic ventilation (MV); to compare the length of stay (LOS), duration of MV, APACHE II and mortality among patients submitted to tracheostomy, according to the moment of its application (early or late).

METHODS: A retrospective observation study type cohort was done from April thru October 2005 including 190 patients at the adult intensive care unit (ICU) of Hospital Estadual do Grajaú.

RESULTS: Among the 190 patients, 32 (16.84\%) were submitted to tracheostomy with a longer LOS $(30.16$ days) as compared to those that were not $(p<0.001)$. The mean time of the tracheostomy procedure was 13.5 days from the starting of the MV. It is known that there still is no guidance for defining an ideal period 
for the operation. On this study, the prevalence of early tracheostomy ( $\leq 13$ days) was $46.87 \%(n=15)$ and the late tracheostomy (> 13 days) was $53.13 \%(n=17)$. In a meaningful way, the patients with early tracheostomy obtained APACHE II superior to those with late tracheostomy (18.2 versus 13.47), however there was no difference regarding the mortality rate. There was no difference regarding the time of ICU LOS (28.9 versus 31.28 days) and the MV time (29.73 versus 32.23 days) for both groups.

CONCLUSIONS: The incidence of tracheostomy was high, being associated to a smaller ICU mortality but with a longer LOS and more complications. There was no significant difference regarding the destination of the patients when submitted to early or late tracheostomy.

Key Words: ICU, mechanical ventilation, tracheostomy

\section{INTRODUÇÃO}

A traqueostomia está entre os procedimentos cirúrgicos mais comumente realizados em pacientes críticos nas unidades de terapia intensiva (UTI) que necessitam de suporte ventilatório prolongado ${ }^{1,2}$.

Na maioria das vezes está indicada nos pacientes com tempo prolongado de ventilação mecânica (VM), no manuseio dos portadores de desmame difícil da prótese ventilatória ou para facilitar a higiene das vias aéreas, oferecendo maior segurança e conforto para o paciente ${ }^{1-8}$, permitindo a retirada do tubo traqueal e a diminuição da sedação durante a VM ${ }^{5}$.

Entretanto, a prática deste procedimento ainda é controversa, devido a inexistência de diretrizes para selecionar qual paciente deve ser submetido a ela, assim como o período ideal para a sua realização ${ }^{1,4,9-11}$. Estes fatores colaboram para que a sua realização seja baseada em aspectos clínicos subjetivos, transformando-a em decisão individual de cada serviço ou do próprio intensivista ${ }^{9,12,13}$.

Diversos estudos têm demonstrado que a traqueostomia precoce encontra-se associada à redução do tempo de VM, de internação na UTI e no hospital, quando comparada com a traqueostomia tardia ${ }^{1,14}$.

O objetivo deste estudo foi estimar, em um centro único, a incidência, o perfil e a evolução dos pacientes submetidos à traqueostomia em relação ao período em que foi realizada; além de comparar o tempo de internação, de VM, escore APACHE II e a mortalidade, entre a traqueostomia precoce e a tardia.

\section{MÉTODO}

Foram avaliados 190 pacientes internados na UTI adulto do Hospital Estadual do Grajaú (HEG), através de estudo retrospectivo observacional (coorte) no período de abril a outubro de 2005.

Dos pacientes que necessitaram de intubação traqueal e/ou traqueostomia, foram analisadas variáveis como idade, sexo, motivo da internação, procedência, escore do Acute Physiology and Chronic Health Evaluation II (APACHE II), risco de óbito segundo esse índice, mortalidade na UTI e na enfermaria, tempo de internação na UTI, valor mínimo de hematócrito e presença de complicações (pneumonia associada à ventilação mecânica, úlceras de decúbito, infecção de corrente sangüínea confirmada por exame laboratorial e infecção sintomática do trato urinário), o intervalo entre a intubação traqueal e a realização da traqueostomia e a duração da ventilação mecânica, através de um protocolo desenvolvido para este estudo.

As complicações foram consideradas quando ocorreram após um período superior a $48 \mathrm{~h}$ da instalação da ventilação mecânica. A pneumonia associada à ventilação foi definida com a presença de nova imagem radiológica, através de revisão radiológica, e/ou mudanças na característica da secreção traqueal.

A traqueostomia precoce foi definida como aquela realizada em período inferior ou equivalente à 13 dias de início da ventilação mecânica e tardia, após 13 dias.

As variáveis estudadas foram descritas utilizando médias e proporções. Os dados coletados foram codificados e transferidos para uma planilha eletrônica, utilizando-se o programa Epilnfo (versão 6.0) ${ }^{15}$.

A análise estatística configurou-se pelo uso do teste do Qui-quadrado ou o teste Exato de Fisher para associação das variáveis qualitativas. Para as variáveis quantitativas utilizou-se o teste de Mann-Whitney ${ }^{16}$. Fixou-se o risco $\leq 0,05$ ou $5 \%$ como nível de rejeição da hipótese de nulidade.

\section{RESULTADOS}

Dos 190 pacientes internados na UTI, 95 (50\%) necessitaram de intubação traqueal associada à ventilação mecânica, sendo que 32 (16,84\%) foram submetidos à traqueostomia no decorrer da internação.

A tabela 1 mostra as características epidemiológicas dos pacientes traqueostomizados. Enquanto a tabela 2 compara as principais variáveis entre os pacientes 
submetidos à intubação traqueal e à traqueostomia. $\mathrm{A}$ tabela 3 compara a presença de complicações entre ambos os grupos.

Tabela 1 - Características Epidemiológicas dos Pacientes Submetidos à Traqueostomia.

\begin{tabular}{lc}
\hline Variáveis & $\mathrm{n}(\%)$ ou Média \pm DP \\
\hline Média etária (em anos) & $50,4 \pm 16,7$ \\
Média do APACHE II & $15,68 \pm 5,9$ \\
Risco de óbito (\%) & $22 \pm 17,7$ \\
Sexo & \\
$\quad$ Feminino & $14(43,75)$ \\
$\quad$ Masculino & $18(56,25)$ \\
Procedência & \\
$\quad$ Clínica médica & $25(78,13)$ \\
$\quad$ Clínica cirúrgica & $7(21,87)$ \\
Motivo da internação & \\
$\quad$ Respiratório & $13(40,62)$ \\
Cardiovascular & $7(21,87)$ \\
Sepse & $5(15,62)$ \\
Pós-operatório & $4(12,5)$ \\
Neurológico & $2(6,25)$ \\
Metabólico/renal & $1(3,12)$ \\
\hline
\end{tabular}

O intervalo médio entre a intubação traqueal e a realização da traqueostomia foi de 13,5 dias (mediana de 13,5), com valores variando entre 8 a 21 dias, obtendo um desvio-padrão equivalente $\mathrm{a} \pm 2,92$ dias.

A tabela 4 demonstra a comparação entre a traqueostomia realizada precocemente (inferior ou igual a 13 dias) e a tardia (superior a 13 dias).

\section{DISCUSSÃO}

A incidência de traqueostomia, segundo alguns autores ${ }^{2,4,12,13,17}$, encontra-se entre $10 \%$ e $11 \%$ dos pacientes internados nas UTI. Neste estudo, obteve-se uma incidência de $16,84 \%$, podendo ser justificado pelo elevado número de internações na UTI por causas respiratórias (40,62\%), uma vez que estas demandam tempo prolongado de $\mathrm{VMI}^{9,18}$.

A baixa faixa etária evidenciada nos pacientes traqueostomizados (50,4 anos) quando comparada a outros estudos $^{4,9}$, pode ter sido influenciada pelas precárias condições sócio-econômicas da região nas proximida-

Tabela 2 - Principais Características Comparativas entre os Pacientes Submetidos à Intubação Traqueal (IT) e à Traqueostomia (TQT).

\begin{tabular}{lccc}
\hline Variáveis & & $\mathrm{n}(\%)$ ou Média \pm DP & Valor-p \\
\hline & Intubação Traqueal & Traqueostomia & \\
Múmero de pacientes & 63 & $50,4 \pm 16,7$ & 0,534 \\
Média etária (em anos) & $47,36 \pm 18,81$ & $18(56,25)$ & 0,934 \\
Sexo & & $14(43,75)$ & $0,001^{*}$ \\
$\quad$ Masculino & $36(57,14)$ & $29,19 \pm 6,39$ & 0,761 \\
$\quad$ Feminino & $27(42,86)$ & $15,68 \pm 5,9$ & 0,354 \\
Média do hematócrito (\%) & $36,26 \pm 9,29$ & $22 \pm 17,7$ & $0,001^{*}$ \\
Média do APACHE II & $20,17 \pm 7,42$ & $30,16 \pm 16,6$ & $0,001^{*}$ \\
Risco de óbito (APACHE II) & $28,16 \pm 21,0$ & $28,87 \pm 18,58$ & $0,001^{*}$ \\
Média de internação na UTI (dias) & $9,8 \pm 7,0$ & & \\
Média de duração da VM (dias) & $7,53 \pm 6,2$ & $25(78,12)$ & $7(21,87)$ \\
Destino do paciente & & $7(21,87)$ & \\
$\quad$ Enfermaria & $24(38,1)$ & $39(61,9)$ & \\
Óbito na UTI & $2(3,2)$ & & \\
$\quad$ Óbito na enfermaria & & & \\
\hline
\end{tabular}

*Valores estatisticamente significativos.

Tabela 3 - Distribuição das Principais Complicações dos Pacientes Submetidos à Intubação Traqueal (IT) e à Traqueostomia (TQT).

\begin{tabular}{lccc}
\hline Variáveis & \multicolumn{1}{c}{} & \\
\hline & Intubação Traqueal & Traqueostomia & Valor-p \\
Pneumonia associada à VM & $15(23,8)$ & $16(50)$ & $0,010^{*}$ \\
Úlcera por pressão & 0 & $5(15,62)$ & $0,036^{*}$ \\
Infecção da corrente sangüínea** & 0 & $4(12,5)$ & $0,011^{*}$ \\
Infecção sintomática do trato urinário & $4(6,35)$ & $8(25)$ & $0,019^{*}$ \\
\hline
\end{tabular}

* Valores estatisticamente significativos.

** Infecção da corrente sangüínea confirmada por exame laboratorial. 
Tabela 4 - Comparação entre Traqueostomia Precoce (inferior ou igual a 13 dias) e Tardia (superior a 13 dias).

\begin{tabular}{|c|c|c|c|}
\hline Variáveis & \multicolumn{3}{|c|}{ n (\%) ou Média \pm DP } \\
\hline Idade (anos) & $53,6 \pm 18$ & $47,59 \pm 15,43$ & 0,406 \\
\hline Feminino & $6(40)$ & $8(47,06)$ & \\
\hline Masculino & $9(60)$ & $9(52,94)$ & \\
\hline Tempo de internação (dias) & $28,9 \pm 18,6$ & $31,28 \pm 15,6$ & 0,610 \\
\hline Escore APACHE II & $18,2 \pm 5,73$ & $13,47 \pm 5,52$ & $0,037^{\star}$ \\
\hline Risco de óbito (APACHE II) & $31,8 \pm 20,7$ & $14,47 \pm 10,37$ & $0,008^{*}$ \\
\hline \multicolumn{4}{|l|}{ Complicações } \\
\hline Pneumonia & $9(60)$ & $7(41,8)$ & 0,288 \\
\hline Alta hospitalar & $8(53,33)$ & $10(58,9)$ & 0,098 \\
\hline
\end{tabular}

* Valores estatisticamente significativos.

des do HEG.

A distribuição por sexo neste estudo foi semelhante ao encontrado por Kollef e col. ${ }^{4}$, no qual obteve-se uma freqüência maior de traqueostomia em pacientes do sexo masculino.

A maioria dos pacientes foi proveniente da clínica médica $(78,13 \%)$, o que se assemelha ao encontrado em outros estudos ${ }^{4,9}$.

Alguns autores ${ }^{4,9,19-21}$, consideraram que a traqueostomia encontra-se associada ao prolongamento da internação nas UTI. No HEG, o tempo médio de internação na UTI dos pacientes submetidos à traqueostomia foi de 30,16 dias, sendo superior, de forma estatisticamente significativa, ao tempo de internação dos pacientes sob intubação traqueal (9,8 dias). Estes resultados podem ser atribuídos ao fato de que os pacientes traqueostomizados foram submetidos a um período maior de ventilação mecânica, como também ao fato de que muitos pacientes submetidos à intubação traqueal tiveram maior taxa de mortalidade, provavelmente por serem mais graves.

De forma estatisticamente significativa, encontrou-se maior mortalidade, na UTI, nos pacientes não submetidos à traqueostomia $(61,9 \%)$, assim como o constatado em outros estudos $2,4,6,9$. No HEG, este dado pode ser justificado pela maior gravidade dos pacientes admitidos que não foram traqueostomizados.

Alguns autores ${ }^{6,9}$ encontraram, nas enfermarias, semeIhante taxa de mortalidade entre ambos os grupos, di- ferentemente deste estudo, no qual a mortalidade dos pacientes traqueostomizados na enfermaria mantevese equivalente à encontrada na UTI, ou seja, mostrando-se superior ao grupo não submetido à traqueostomia. Essa baixa taxa de mortalidade dos pacientes com traqueostomia nos outros estudos, pode ser decorrente da possibilidade e da facilidade de transferência dos pacientes com prognósticos reservados para outros setores dos hospitais, como as unidades semiintensivas ${ }^{9,22}$.

A média dos menores valores de hematócrito evidenciados nesses pacientes foi inferior de forma estatisticamente significativa no grupo submetido à traqueostomia. Este achado pode ser justificado pela relação direta entre o seu valor e o da hemoglobina, importante carreador de oxigênio para os tecidos, podendo dificultar, dessa forma, a oxigenação tecidual e conseqüentemente o desmame ventilatório.

A traqueostomia pode ser responsável pela elevação na taxa de complicações, como a pneumonia associada à ventilação mecânica e a sepse $e^{4,9,22,23}$. Neste estudo a incidência de pneumonia foi estatisticamente significativa maior nos pacientes traqueostomizados (50\%). Isso pode ser devido ao maior período de ventilação mecânica necessitado por esse grupo, como também ao maior tempo de internação na UTI, ambiente propicio para a contrair novas infecções.

Além das infecções de vias aéreas inferiores, a presença de úlceras de decúbito, infecção sintomática do 
trato urinário e infecção da corrente sangüínea confirmada por exame laboratorial também foram mais freqüentemente encontradas nos pacientes submetidos à traqueostomia.

Todos esses fatores encontram-se também, intimamente ligados, ao aumento significativo do tempo de internação, dos custos hospitalares e da maior morbidade dos pacientes que necessitaram de traqueostomia ${ }^{22}$.

É importante ressaltar que nenhuma das desvantagens descritas faz com que a traqueostomia seja menos aceita do que qualquer outro procedimento habitualmente realizado em pacientes críticos, sendo essencial em algumas ocasiões ${ }^{4,10}$.

No presente estudo foi encontrada uma média de 13,5 dias entre a realização da intubação traqueal e da traqueostomia, período muito semelhante ao evidenciado por Frutos-Vivar e col. (12 dias) ${ }^{9}$.

O tempo ideal para realização da traqueostomia ainda é um assunto controverso. O VIII Consenso da Sociedade Francesa de Medicina Intensiva e o Consenso Norte Americano de 1986 propõem a realização da traqueostomia precoce em pacientes nos quais se prevê o uso de ventilação mecânica por período superior a 21 dias e desencorajada, se este período for inferior a 10 dias. No entanto, a maior dificuldade em aplicar essa recomendação é a dificuldade em se prever a duração da ventilação mecânica .

Recentes estudos mostraram benefícios significativos da traqueostomia precoce em termos de melhora dos resultados clínicos, incluindo redução na duração da ventilação mecânica, internação na UTI e na pneumonia adquirida, associada à ventilação mecânica, sendo a diminuição de sedação e a mobilidade precoce do paciente as principais responsáveis por esses resultados $^{1,7,23-27}$. No entanto, muitas questões ainda não foram respondidas, como qual seria a definição de traqueostomia precoce, se aquela realizada com 2, 7 ou 10 dias de ventilação mecânica ${ }^{5}$, ou quais seriam os pacientes que melhor se beneficiariam dessa técnica ${ }^{9}$. Neste estudo, não foi observada diferença estatisticamente significativa em relação ao tempo de internação, VM, morbidade e mortalidade entre as duas técnicas ${ }^{28}$.

A maioria dos autores atribui à traqueostomia a capacidade de promover melhor conforto ao paciente crítico, quando comparada à intubação traqueal, mas isto não tem sido bem investigado. Ela é responsável, também, pela diminuição da resistência das vias aéreas e do trabalho ventilatório, mas os estudos desenvolvidos para esclarecer suas vantagens e desvantagens possuem muitas limitações, restringindo as conclusões acerca de sua realização. Quanto ao período ideal para sua realização, ainda não existe consenso sobre como sua realização precoce ou tardia pode afetar na duração do suporte ventilatório ${ }^{9,14}$.

Todos estes fatores, em conjunto, enfatizam a necessidade de novos estudos para o embasamento científico em relação a este tema, adequando as indicações desse procedimento e o tempo ideal para sua realização.

\section{CONCLUSÃO}

A incidência de traqueostomia na UTI do HEG encontra-se acima daquela relatada na literatura, sendo o período de sua realização equivalente ao referido em outros estudos.

Não foram observadas diferenças estatisticamente significativas quanto ao sexo, a faixa etária e o escore APACHE II, entre os pacientes traqueostomizados e os intubados. Entretanto, os pacientes submetidos à traqueostomia apresentaram maior tempo médio de permanência na UTI, maior taxa de mortalidade nas enfermarias, maior incidência de pneumonia associada à ventilação mecânica, úlceras de decúbito, infecções sangüíneas e do trato urinário.

Quanto ao período de realização da traqueostomia, apesar de muitos autores demonstrarem que a traqueostomia precoce encontra-se associada à redução do tempo de ventilação mecânica e do tempo de internação na UTI. Neste estudo estas vantagens não foram evidenciadas.

\section{REFERÊNCIAS}

01. Freeman BD, Borecki IB, Coopersmith CM et al - Relationship between tracheostomy timing and duration of mechanical ventilation in critically ill patients. Crit Care Med, 2005;33:2513-2520.

02. Kollef $\mathrm{MH}$ - Tracheostomy for respiratory failure: we need more answers. Chest, 2004;125:7-9.

03. Angel LF, Simpson CB - Comparison of surgical and percutaneous dilational tracheostomy. Clin Chest Med, 2003;24:423-429.

04. Kollef MH, Ahrens TS, Shannon W - Clinical predictors and outcomes for patients requiring tracheostomy in the intensive care unit. Crit Care Med, 1999;27:1714-1720.

05. Ahrens T, Kollef $\mathrm{MH}$ - Early tracheostomy--has its time arrived? Crit Care Med, 2004;32:1796-1797.

06. Blot F, Melot C - Indications, timing and techniques of tracheostomy in 152 French ICUs. Chest, 2005;127:1347-1352.

07. Heffner JE - Timing of tracheotomy in mechanically ventilated patients. Am Rev Respir Dis, 1993;147:768-771.

08. Heffner JE - Timing tracheotomy: calendar watching or individualization of care? Chest, 1998;114:361-363.

09. Frutos-Vivar F, Esteban A, Apezteguia C et al - Outcome of mechanically ventilated patients who require a tracheostomy. Crit Care Med, 2005;33:290-298.

10. Heffner JE - The role of tracheotomy in weaning. Chest, 2001;120:(Suppl6)477S-481S. 


\section{ESTUDO COMPARATIVO ENTRE TRAQUEOSTOMIA PRECOCE E TARDIA EM PACIENTES SOB VENTILAÇÃO MECÂNICA}

11. Heffner JE - Tracheotomy application and timing. Clin Chest Med, 2003;24:389-398.

12. Esteban A, Anzueto A, Alia I et al - How is mechanical ventilation employed in the intensive care unit. An international utilization review. Am J Respir Crit Care Med, 2000;161:1450-1458.

13. Fischler L, Erhart S, Kleger GR et al - Prevalence of tracheostomy in ICU patients. A nation-wide survey in Switzerland. Intensive Care Med, 2000;26:1428-1433.

14. Ferguson ND - Tracheostomy for ventilated patients--not when, but whom? Crit Care Med, 2005;33:2695-2696.

15. Epilnfo: Database and Statistics Program for Public Health Professionals. [computer program]. Version 6.0. Atlanta (Georgia-USA): Center for Disease Control Prevention; 2006.

16. Siegel S, Castellan NJ Jr - Non Parametric Statistics, em: McGraw-Hill International Editions, $2^{\text {nd }}$ Ed. New York: McGraw-Hill Press; 1988.

17. Cox CE, Carson SS, Holmes GM et al - Increase in tracheostomy for prolonged mechanical ventilation in North Carolina, 1993-2002. Crit Care Med, 2004;32:2219-2226.

18. Lam S, Ridley S - Critically ill medical patients, their demographics and outcome. Anaesthesia, 1999;54:845-852.

19. Engoren M, Arslanian-Engoren C, Fenn-Buderer N - Hospital and long-term outcome after tracheostomy for respiratory failure. Chest, 2004;125:220-227.
20. Kurek CJ, Cohen IL, Lambrinos J et al - Clinical and economic outcome of patients undergoing tracheostomy for prolonged mechanical ventilation in New York State during 1993: analysis of 6,353 cases under diagnosis-related group 483. Crit Care Med, 1997;25:983-988.

21. LoCicero $\mathrm{J} 3^{\text {rd }}$, McCann $B$, Massad $M$ et al - Prolonged ventilatory support after open-heart surgery. Crit Care Med, 1992;20:990-992.

22. Warren DK, Shukla SJ, Olsen MA et al - Outcome and attributable cost of ventilator-associated pneumonia among intensive care unit patients in a suburban medical center. Crit Care Med, 2003;31:1312-1317.

23. Wright SE, VanDahm K - Long-term care of the tracheostomy patient. Clin Chest Med, 2003;24:473-487.

24. Rana S, Pendem S, Pogodzinski MS et al - Tracheostomy in critically ill patients. Mayo Clin Proc, 2005;80:1632-1638.

25. Brook AD, Sherman $\mathrm{G}$, Malen $\mathrm{J}$ et al - Early versus late tracheostomy in patients who require prolonged mechanical ventilation. Am J Crit Care, 2000;9:352-359.

26. Perren A - Further clarification? Crit Care Med, 2005;33:466-467.

27. Moller MG, Slaikeu JD, Bonelli $P$ et al - Early tracheostomy versus late tracheostomy in the surgical intensive care unit. Am J Surg, 2005;189:293-296.

28. Sinuff T-Review: early tracheostomy is not better than late tracheostomy for reducing all - cause mortality in critically ill patients. ACP Club, 2005;143:62. 